achtungen gestützt, dass ein höherer Gebalt der Lösung an Ammoniumnitrat das Ausfällen der Phosphormolybdänverbindung befördert.

Die Lösung des gelben Niederscblages in Ammoniak soll sofort und vollkommen klar sein. Das trifft wohl in den meisten Fällen ein, wenn aber nicht, so ist es eine Quelle von Unannehmlichkeiten.

Nach Wagner (Landw. Vers. 45, 384) ist die Phosphorsäurebestimmung nach der Molybdänmethode auszufübren, da bei der Citratmethode die Kieselsäure störend wirkt. Über den Kieselsäuregehalt der Lösungen und dessen Einfluss auf die Fällung sollen auch später näbere Angaben folgen. Nach unseren Ermittlungen werden nach der Citratmethode bei Verwendung von 60 cc Maerckerscher Citratlösung und 45 cc Magnesiamixtur auf 50 cc Lösung (entsprechend $0,5 \mathrm{~g}$ Substanz) Resultate erhalten, die mit denen der Molybdänmethode in der Regel sebr gut übereinstimmen, in seltenen Fällen aber ein klein wenig niedriger sind, was jedoch in der Gegenwart von Kieselsāure keinesfalls seinen Grund hat. (Belegzahlen folgen.)

Nachdem jedoch die Citratmethode nur in ihrer Anwendung für die Bestimmung der wasserlöslichen Phosphorsāure der Superphosphate durchaus verlässliche Resultate liefert, wie ich seinerzeit (d. Z. 1889, 702; 1890,19 u. 196) nachzuweisen versuchte, wäre sie für die Bestimmung der citratlöslichen Phosphorsäure in den Thomasschlacken nur dann ohne weiteres zu verwenden, wenn es sich um die früher vorgeschlagene Classifikation oder Gütebestimmung handelt. Soll die "citratlösliche" als Werthbasis gelten, dann könnten gar nicht genug Vergleichsbestimmungeu an verschiedenen Orten gemacht und veröffentlicht werden, bevor man die Methode zur allgemeinen Annahme empfiehlt.

Was die Einwirkungsdauer der Citratlösung auf das Phosphat betrifft, so könnte es nach den Mittheilungen von Gerlach und Passon (a. a. O.) scheinen, dass ein Abweichen von der Wagner'schen Vorschrift, genau 30 Minuten rotiren zu lassen, recht geringfügige Differenzen verursacht, die sogar bei wesentlicher Überschreitung der vorgeschriebenen Zeit kaum in's Gewicht fallen.

Dies trifft nach meinen Erfabrungen in einzelnen Fällen auch wirklich zu; bei manchen Schlacken aber gibt eine länger fortgesetzte Digestion erheblich böhere Resultate, so dass unter Umständen eine Ungenauigkeit von 5 bis 10 Minuten bemerkbare Fehler bedingen kann. Stundenlang fortge- setzte Digestion gibt in anderen Fällen wieder eine auffallend niedrigere Ausbeute an gelöstem Phosphat, so dass man ein Unlöslichwerden schon gelöster Phosphate dabei annehmen muss.

Möglicherweise spielt das metallische Eisen, das in Schlacken ja nie ganz fehlt, dabei eine Rolle. Ausreichende Erklärungen für alle diese Lösungsvorgänge sind ja heute noch nicht möglich.

Schliesslich möchte ich nicht unerwähot lassen, dass der als Pilzgift so viel gepriesene Formaldehyd sich mir seit Jahren als sicheres Mittel bewährt hat, die so sehr zum Schimmeln geneigten verdünnten Lösungen von Ammoncitrat und freier Citronensãure vor dem Verderben zu bewahren.

Während reine Lösungen schon nach 6 bis 8 Tagen Pilzvegetationen zeigen, bleiben Lösungen, denen auf $15 l$ etwa 5 cc Formalin Schering (ungefähr 40 Proc. Formaldehyd enthaltend) zugesetzt sind, unbegrenzt haltbar.

Nachdem jetzt schon in den Versuchsstationen und anderen Laboratorien jährlich Tausende von Bestimmungen der citratlöslichen Phosphorsäure in Thomasschlacken vorgenommen werden, erscheint die allgemeine Prüfung zunächst der folgenden Fragen ein dringendes Bedürfniss:

1. Ist die Anwendung der Molybdänmethode dabei nothwendig?

2. Könnte nicht zweckmässig die Wagner'sche Lösung durch die Gerlach'sche ersetzt werden?

Nach den diesseitigen Erfahrungen dürften beide Fragen zu Gunsten einer schnelleren, sicheren und einfacheren Ausführbarkeit beantwortet werden, worüber demnächst. weitere Mittheilungen folgen.

K. k. landwirthschaftlich-chemische Versuchsstation Wien.

\section{Nachtrag zu der Abhandlung}

von Mann, Krüger und Tollens über

Bestimmung der Pentosen und Pentosane durch Furfuroldestillation.

$$
\text { Von }
$$

\section{B. Tollens.}

In meiner Abhandlung auf S. 33 des 2. Heftes dieses Jahrganges dieser Zeitschrift habe ich zur Umrechnung des Furfurols, welches man beim Destilliren pentosanhaltiger Pflanzenstoffe mit Salzsäure gewinnt, auf Pentosan nicht die ron Mann und mir früher benutzten, sondern einige neue Formeln angegeben, welche den Aus- 
druck ("Furfurol - 0,0104") enthalten, und welche ich aus der zur Umrechnung des Hydrazonniederschlages auf Furfurol benutzten Formel (S. 35)

Hydrazon $\times 0,516+0,0104=$ Furfurol herleitete.

Mir sind nun Bedenken darüber aufgestiegen, ob es gut ist, bei einer conventionellen Methode, welche ibrer Natur nach doch nicht immer absolut richtige Resultate liefert, und bei welcher man nicht alle Einfluss habenden Momente immer genügend beurtheilen kann, verschiedene Formeln zu geben oder die anzuwendenden Formeln irgendwie zu compliciren, und ich möchte deshalb vorschlagen, zu dem von Mann und mir früher gegebenen einfachen Factor 1,84 der Umrechnung von Furfurol auf Pentosan zurückzukehren, und also die früher gegebenen Formeln und besonders den Ausdruck

$$
\text { Furfurol } \times 1,84=\text { Pentosan }
$$

im Allgemeinen beizubebalten, welchen u. a. Stift schon benutzt hat.

Der Unterschied in den Resultaten gegenüber denen der Formel

(Furfurol - 0,0104) $\times 1,88=$ Pentosan i. A. ist meistens gering und verschwindend gegenüber den Differenzen, welche durch das Vorhandensein von mehr oder weniger Pentosan in den mit Salzsäure destillirten Vegetabilien und durch andere Umstände bewirkt werden können.

Ich schlage demnach vor, die Pentosanbestimmung durch Destillation mit Salzsäure, die Fällung des Furfurols durch Fällung mit Phloroglucin, und weiter die Berechnung des erbaltenen Furfurols auf Pentosan mittels des Ausdrucks

Furfurol $\times 1,84=$ Pentosan im Allgemeinen zu bewirken.

Glaubt man, dass nur Xylan oder nur A raban in der untersuchten Pflanzensubstanz vorhanden sind, kann man den Ausdruck

$$
\begin{aligned}
& \text { Furfurol } \times 1,64=\text { Xylan } \\
& \text { Furfurol } \times 2,02=\text { Araban }
\end{aligned}
$$

anwenden, doch weiss man obiges selten gewiss, und es wird der Gleichförmigkeit halber besser sein, den Durchschnittsfactor 1,84 für Pentosan im Allgemeinen zu verwenden.
Über einige neue Laboratoriums-Apparate. Von

Max Kaehler \& Martini, Berlin.

1. Apparate zu Schmelzpunktsbestimmungen. Es ist sehr bequem und praktisch, im Laboratorium einen speciell zu Schmelzpunktsbestimmungen stets fertigen Apparat zur Verfügung zu haben. Herr Prof. Dr. C. A. Bischoff liess zu diesem Zweck den Apparat Fig. 68 anfertigen, der sich seit längerer Zeit bewährt hat. Das Stativ hat eine feste eiserne Platte und einen Aluminiumstab, zwei Aluminium-

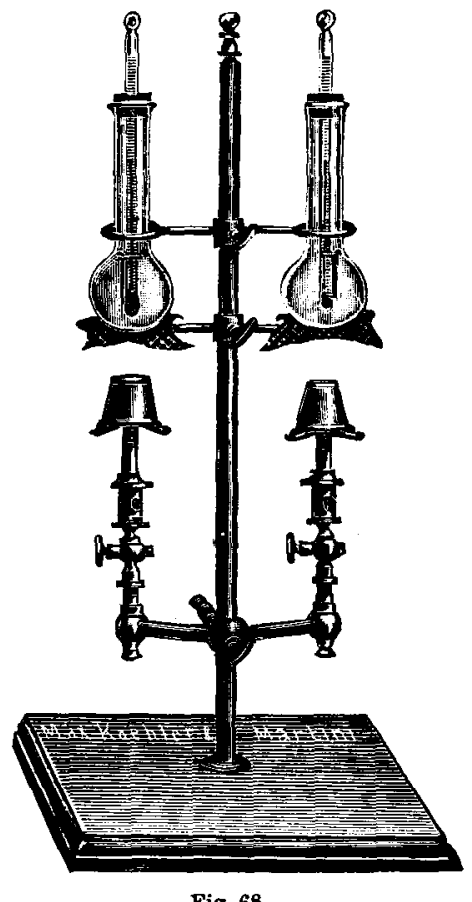

Fig. 68.

doppelringe und einen Doppelbrenner, ebenfalls aus Aluminium. Die Schmelzpunktkölbchen werden durch die Ringe sicher gehalten und ruhen auf Asbestdrahtnetzen. Das eine Kölbchen wird mit Wasser, das andere mit Olivenöl gefüllt. Die Apparate können für niedrig- (Wasser) und hochschmelzende (Olivenöl) Substanzen gebraucht werden und sind immer zum Gebrauche fertig.

2. Apparate zum Chloriren und Bromiren an der Sonne. Herr Prof. Dr. Schramm bedient sich seit einiger Zeit der Erlenmeyer-Kochflaschen, weil in diesen die Sonnenstrahlen die Glaswände ziemlich senkrecht treffen und viel weniger zerstreut werden als - beim Gebrauch runder Kochflaschen. Praktisch bewäbren sich diese ganz aus Glas hergestellten Apparate, da die Berührung von Chlor und Brom mit 